\title{
A Fading Filter Design for Multipath Rayleigh Fading Simulation and Comparisons to Other Simulators
}

\author{
Ali Arsal \\ Department of Electrical and \\ Electronics Engineering \\ İzmir Institute of Technology \\ Gülbahçe, 35430, Urla, İzmir, Turkey \\ Email: aliarsal@iyte.edu.tr
}

\author{
Serdar Özen \\ Department of Electrical and \\ Electronics Engineering \\ Izmir Institute of Technology \\ Gülbahçe, 35430, Urla, İzmir, Turkey \\ Email: serdarozen@iyte.edu.tr
}

\begin{abstract}
A low-complexity high performance Rayleigh fading simulator, an ARMA $(3,3)$ model, is proposed. This proposed method is a variant of the method of filtering of the white Gaussian noise where the filter design is accomplished in the analog domain and transferred into digital domain. The proposed model is compared with improved Jakes' model, autoregressive filtering and IDFT techniques, in performance and computational complexity. Proposed method outperforms AR(20) filter and modified Jakes' generators in performance. Although IDFT method achieves the best performance, it brings a significant cost in storage and is undesirable. The proposed method achieves high performance with the lowest complexity.
\end{abstract}

\section{INTRODUCTION}

The fading caused by multipath propagation in wireless communication systems is commonly modelled by the Rayleigh distribution. It is well known that a Rayleigh fading process is characterized by its power spectral density and its auto-correlation function. The correlation function depends on the Doppler frequency which corresponds to the relative motion of the receiver and transmitter. In the communications literature, a number of different methods have been proposed and used for the simulation of Rayleigh fading. Jakes' model [1] has been of great interest which is based on sum of sinusoids approach. Simulators based on white noise filtering methods [2], [3] and on the Inverse Discrete Fourier Transform (IDFT) method [4], [5] have also become popular. It was shown in [6] that the fading signals which are produced by classical Jakes' simulator are not wide-sense stationary (WSS). On the other hand simulators based on the IDFT method are of high-quality and efficient. Unfortunately, a disadvantage of the IDFT method is that all samples are generated with a single fast Fourier transform (FFT), hence the storage requirements make it useless for the generation of very large number of samples and for sample-by-sample simulations.

In this paper, we consider using a fading filter to filter white Gaussian noise that was first proposed in [2]. Unlike the other filter structures [1], [3], [4], [5], [7], a different optimization/design criterion is used to set the filter parameters in the analog domain as would yield the transfer function of the fading filter, denoted by $G_{\gamma}(s)$, where $\gamma$ is the filter order. Bilinear transform is then used to get the desired filter structure as an $\operatorname{ARMA}(\gamma, \gamma)$ filter. Comparisons to other methods are then made by using quantitative measures introduced in [8].

\section{RAYLEIGH FADING STATISTICS}

Rayleigh fading process is characterized by the Gaussian WSS uncorrelated scattering fading model [9], where the fading process is modelled as a complex Gaussian process. In this model, time variability of the channel is determined by its autocorrelation function. This statistic generally depends on the propagation geometry, the velocity of the mobile and the antenna characteristics. A common assumption is that the propagation path consists of two-dimensional isotropic scattering with a vertical monopole antenna at the receiver [1]. In this case theoretical spectral density of the in-phase or quadrature part of the received signal is represented as

$$
S(f)=\left\{\begin{array}{cl}
\frac{\sigma^{2}}{2 \pi f_{d} \sqrt{1-\left(f / f_{d}\right)^{2}}} & |f| \leq f_{d} \\
0 & \text { else }
\end{array}\right.
$$

where $\sigma^{2}$ is the rms value of the envelope of the waveform, or equivalently it is the fractional power of each lag. $f_{d}$ is the maximum Doppler shift which is defined as the ratio of the vehicle speed, $V$, to the wavelength, $\lambda, f_{d}=V / \lambda$, and $\lambda=$ $c / f_{c}$ where $c=3 \times 10^{8} \mathrm{~m} / \mathrm{sec}$ is the speed of the light, and $f_{c}(\mathrm{~Hz})$ is the carrier frequency. The corresponding normalized (unit-variance) continuous time autocorrelation function of the received signal under these conditions is $R(\tau)=J_{0}\left(2 \pi f_{d}|\tau|\right)$, where $J_{0}(\cdot)$ is the zeroth-order Bessel function of the first kind. For the discrete-time simulation of this model, ideally generated in-phase and quadrature Gaussian processes should each have the autocorrelation sequence

$$
R[n]=J_{0}\left(2 \pi f_{m}|n|\right)
$$

where $f_{m}=f_{d} T$ is the maximum Doppler frequency normalized by the sampling rate $1 / T$. Furthermore, in this model the 
in-phase and quadrature processes must be independent and each must have zero mean for Rayleigh fading.

\section{DERIVATION OF THE FADING FILTER}

A straightforward method to simulate a faded signal is to amplitude modulate the carrier signal with a low-pass filtered Gaussian noise source as shown in Figure 1. In order to obtain

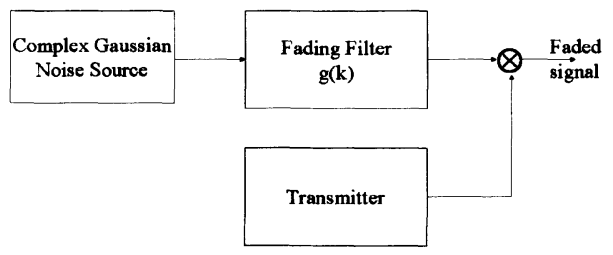

Fig. 1. Faded signal generator that uses low-pass filtered white complex Gaussian noise

time varying frequency selective fading channel we must have a bank of these fading filters where each filter generates the corresponding fading channel tap. A fading filter with impulse response $g(k)$ can be designed so that its output spectral density is an approximation to theoretical spectral density of the complex envelope of the faded signal $S(f)$. We will use filter structures that were proposed in [2]. Consider the elementary first order filter transfer function $G_{1}(s)$, and the second order filter transfer function $G_{2}(s)$ where

$$
G_{1}(s)=\frac{w_{x}}{s+w_{x}}
$$

and

$$
G_{2}(s)=\frac{w_{x}^{2}}{s^{2}+\frac{w_{x} s}{Q}+w_{x}^{2}} .
$$

Then we can have fading filter continuous time transfer functions with higher orders (of order $\gamma$ ), $G_{\gamma}(s)$, that are given by

$$
G_{\gamma}(s)=\left\{\begin{array}{cl}
G_{2}^{\gamma / 2}(s), & \text { if } \gamma \text { even } \\
G_{1}(s) G_{2}^{(\gamma-1) / 2}(s), & \text { if } \gamma \text { odd }
\end{array}\right.
$$

where $G_{1}(s)$ and $G_{2}(s)$ are as given by (3) and (4) respectively, and the selection of $Q$ is such that there is a pre-specified frequency response level at $w=w_{x} \mathrm{rad} / \mathrm{sec}$; for example for the third-order filter if $Q=\sqrt{10}$ then the magnitude of $G(\cdot)$ will have a gain of $7 \mathrm{~dB}$ at $w=w_{x}(10 \mathrm{~dB}$ gain from the second order filter and $-3 \mathrm{~dB}$ from the first order part making the overall gain of $7 \mathrm{~dB}$ ). In order to find the parameters of the fading filter transfer function, $G_{\gamma}(s)$, we will first set the filter order $\gamma$ and $Q$. Then defining $S(f ; \epsilon)$, as an approximation to the theoretical spectral density of (1), by

$$
S(f ; \epsilon)=\left\{\begin{array}{cl}
\frac{\sigma^{2}}{2 \pi f_{d} \sqrt{1-\left(f / f_{d}\right)^{2}}} & |f| \leq f_{d}-\epsilon \\
0 & \text { else }
\end{array}\right.
$$

TABLE I

RATIO OF $w_{x} / w_{d}$ TABULATED WITH RESPECT TO VARIOUS FILTER ORDERS AND DESIRED PEAK $(d B)$ AT $w_{x}=w_{d}$

\begin{tabular}{|c||c|c|c|}
\hline \multicolumn{1}{|c||}{ Filter Order } & \multicolumn{3}{c|}{ Desired Peak $(d B)$ at $w=w_{x}$} \\
\hline$\gamma$ & $\mathbf{1 0}$ & $\mathbf{1 5}$ & $\mathbf{2 0}$ \\
\hline $\mathbf{2}$ & 1.0200 & 1.0055 & 1.0025 \\
\hline $\mathbf{3}$ & 1.0152 & 1.0060 & 1.0017 \\
\hline $\mathbf{4}$ & 1.0668 & 1.0401 & 1.0247 \\
\hline $\mathbf{5}$ & 1.0668 & 1.0413 & 1.0228 \\
\hline
\end{tabular}

where $\epsilon \in \mathbf{R}^{+}$is a small positive real number, which can be taken as multiples of the smallest positive number the computing platform that can handle. Then we solved the numerical optimization problem, for fixed $\gamma, f_{d}$ and $Q$,

$$
w_{x}=\arg \min \left\|S(f ; \epsilon)-\left|G_{\gamma}(j 2 \pi f)\right|^{2}\right\| .
$$

The result of this numerical optimization (7) gives the minimizer of the norm of the distance between the modified theoretical spectral density and the theoretical fading filter spectrum. Theoretical and approximate spectral density, where the approximate spectral density is for the output of the filter $G_{3}(s)$, are provided in Figure 2. For the transfer functions provided in the $s$-domain, we can use the bilinear transform to get $G_{\gamma}(z)$ with an $\operatorname{ARMA}(\gamma, \gamma)$ model, or impulse invariance method to get a $G_{\gamma}(z)$ with an $\operatorname{AR}(\gamma)$ model (all pole filter), where

$$
G_{\gamma}(z)=\frac{\sum_{k=0}^{\gamma} g_{k}^{M} z^{-k}}{1-\sum_{k=1}^{\gamma} g_{k}^{A} z^{-k}}
$$

with $\left\{g_{k}^{A}\right\}_{k=1}^{\gamma},\left\{g_{k}^{M}\right\}_{k=0}^{\gamma}$ are the auto-regressive and movingaverage filter taps, of the $\operatorname{ARMA}(\gamma, \gamma)$ model, respectively.

The generated Rayleigh fading process has an autocorrelation function, $R_{x x}[k]$, which can be found by directly using Wiener-Khinchine theorem [10]. That is,

$$
R_{x x}[k]=\sigma^{2} g[k] * g[-k]
$$

where $\sigma^{2}$ is the variance of the complex zero-mean white Gaussian noise, and $g[k]=\mathcal{Z}^{-1}\left(G_{\gamma}(z)\right)$ is the discrete time filter impulse response and as given as the inverse $\mathcal{Z}$-transform of the transfer function $G_{\gamma}(z)$.

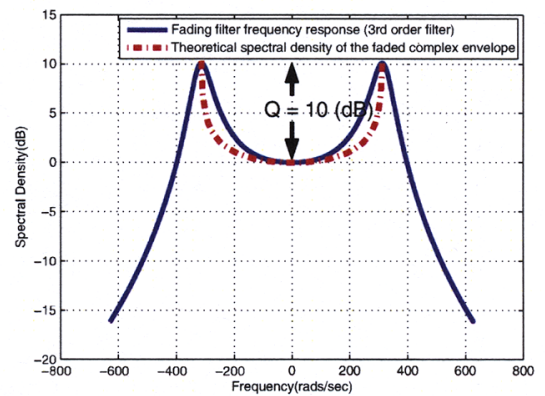

Fig. 2. Theoretical and approximate spectral density (for the filter $G_{3}(s)$ ) 


\section{PERformance AND Complexity Evaluation}

In this section, we evaluate the suitability of our proposed filter design technique for producing high-quality Rayleigh fading sequence. Comparisons of our proposed method provided in Section 3 are made to a WSS-improved Jakes' model of [7], AR fading filter approximation of [3], and to the IDFT technique which was shown in [5] to be the most efficient and highest quality method among different Rayleigh fading generator design methods. First, the quantitative measures that are used for this comparison are described.

\section{A. Quantitative Measures}

Quantitative quality measures for generated random sequences have been proposed in [8]. Two quality measures have been defined in [8] as follows. The first measure, called the mean basis power margin, is given by

$$
\mathcal{G}_{\text {mean }}=\frac{1}{\sigma_{X}^{2} L} \operatorname{trace}\left\{C_{X} C_{\hat{X}}^{-1} C_{X}\right\}
$$

and the second measure, the maximum basis power margin, is defined as

$$
\mathcal{G}_{\max }=\frac{1}{\sigma_{X}^{2}} \max \left\{\operatorname{diag}\left\{C_{X} C_{\hat{X}}^{-1} C_{X}\right\}\right\}
$$

In (10) and (11), $\sigma_{X}^{2}$ is the variance of the reference(ideal) distribution, $C_{\hat{X}}$ is the $L \times L$ covariance matrix of any length- $\mathrm{L}$ subset of adjacent samples produced by the stationary random sequence generator, and $C_{X}$ represents the desired covariance matrix of $L$ ideally distributed samples.

\section{B. Tested Simulation Methods}

1) Our Proposed Filter Design Method: Our filter design was accomplished in the analog domain and transferred into the digital domain and implemented via ARMA model or AR model by bilinear transform using the MATLAB function bilinear, or impulse invariance method by MATLAB function impinvar respectively. After the filter coefficients were calculated, the Rayleigh fading sequence was generated by a direct structure using the MATLAB function filter.

2) IDFT Method: The simulator used was implemented as described in [5]. In this method, the IDFT operation is applied to complex sequences of independent, normally distributed random numbers, each sequence multiplied by suitable filter coefficients. The filter coefficients can be determined by equation (12), where $\mathrm{N}$ denotes the number of symbols and $k_{m}=\left\lfloor\left(f_{m} N\right)\right\rfloor$ The MATLAB function ifft was used for IDFT computation.

3) AR Method: The method used was that of [3].The basic relationship between the desired model autocorrelation function $R_{x x}[k]$ and the $\mathrm{AR}(\mathrm{p})$ parameters is given by:

$$
R_{x x}[k]= \begin{cases}-\sum_{m=1}^{p} a_{m} R_{x x}[k-m], & k \geq 1 \\ -\sum_{m=1}^{p} a_{m} R_{x x}[k-m]+\sigma_{p}^{2}, & k=0 .\end{cases}
$$

In the matrix form this becomes for $k=1,2, \cdots, p$

$$
\mathbf{R}_{\mathbf{x x}} \mathbf{a}=-\mathbf{v}
$$

where

$$
\begin{aligned}
& \mathbf{R}_{\mathbf{x x}}=\left[\begin{array}{cccc}
R_{x x}[0] & R_{x x}[-1] & \cdots & R_{x x}[-p+1] \\
R_{x x}[1] & R_{x x}[0] & \cdots & R_{x x}[-p+2] \\
\vdots & \vdots & \ddots & \vdots \\
R_{x x}[p-1] & R_{x x}[p-2] & \cdots & R_{x x}[0]
\end{array}\right], \\
& \mathbf{a}=\left[a_{1}, a_{2}, \cdots, a_{p}\right]^{T}, \mathbf{v}=\left[R_{x x}[1], R_{x x}[2], \cdots, R_{x x}[p]\right]^{T}, \\
& \sigma_{p}^{2}=R_{x x}[0]+\sum_{k=1}^{p} a_{k} R_{x x}[k] .
\end{aligned}
$$

Given the desired autocorrelation sequence, the AR filter coefficients can be determined by solving the set of $p$ YuleWalker equations. These equations can in principle be solved by the Levinson-Durbin recursion. The method of [3] was implemented via MATLAB function filtic, to generate first $p$ (model order) stationary Rayleigh fading samples and then MATLAB function filter was used to generate the other samples.

4) WSS-improved Jakes' Model: The method used was based on the sum of sinusoids technique of [7]. The normalized low-pass discrete fading process is generated by

$$
\begin{aligned}
x[n] & =x_{c}[n]+j x_{s}[n], \\
x_{c}[n] & =\frac{1}{\sqrt{N_{s}}} \sum_{k=1}^{N_{s}} \cos \left(2 \pi f_{m} n \cos \alpha_{k}+\phi_{k}\right) \\
x_{s}[n] & =\frac{1}{\sqrt{N_{s}}} \sum_{k=1}^{N_{s}} \cos \left(2 \pi f_{m} n \sin \alpha_{k}+\varphi_{k}\right)
\end{aligned}
$$

with

$$
\alpha_{k}=\frac{2 \pi k-\pi+\theta}{4 N_{s}}, \quad k=1,2, \cdots, N_{s}
$$

where $\phi_{k}, \varphi_{k}$ and $\theta$ are statistically independent and uniformly distributed on $[-\pi, \pi)$ for all k. For finite $N_{s}$, this WSS simulator is not autocorrelation ergodic. So, theoretical calculations of quality measures can't be done for this method.

\section{Performance Comparisons}

The quality measure comparison results, which are presented in Table II, compare the quality of the real part of the simulator outputs. Similar results were achieved for the imaginary sequences and these are omitted for brevity. Perfect Rayleigh fading sequence generation method corresponds to $0 \mathrm{~dB}$ for both measures. In all cases, the reference autocorrelation function is (2) with a normalized maximum Doppler of $f_{m}=0.05$. An autocorrelation sequence length of 200 was considered for evaluation of all theoretical ( $T$ ) results. For the empirical (E) results, time average correlations were calculated based on $2^{20}$ generated samples. The computed quality measures were then averaged over 50 independent simulation trials. Plots of the empirical autocorrelation functions of the AR model and our proposed Rayleigh fading generator via $A R$ models are shown in Figure 3 and the plots of the IDFT method and our proposed filter generator via ARMA models are shown in Figure 4 . The results show that the 


$$
F[k]=\left\{\begin{array}{cl}
0 & k=0, \\
\sqrt{\frac{1}{2 \sqrt{1-\left(\frac{k}{N f_{m}}\right)^{2}}}} & k=1,2, \ldots, k_{m}-1, \\
\sqrt{\frac{k_{m}}{2}\left[\frac{\pi}{2}-\arctan \left(\frac{k_{m}-1}{\sqrt{2 k_{m}-1}}\right)\right]} & k=k_{m}, \\
0, & k=k_{m}+1, \ldots, N-k_{m}-1, \\
\sqrt{\frac{k_{m}}{2}\left[\frac{\pi}{2}-\arctan \left(\frac{k_{m}-1}{\sqrt{2 k_{m}-1}}\right)\right]} & k=N-k_{m}, \\
\sqrt{\frac{1}{2 \sqrt{1-\left(\frac{N-k}{N f_{m}}\right)^{2}}}} & k=N-k_{m}+1, \ldots, N-1 .
\end{array}\right.
$$

TABLE II

QUALITY MEASURES FOR THE IDFT, OUR PROPOSED FILTER DESIGN VIA AR AND ARMA, AR FILTERING AND SUM OF SINUSOIDS METHODS OF GENERATING BANDLIMITED RAYLEIGH RANDOM SEQUENCES FOR COVARIANCE SEQUENCE LENGTH 200

\begin{tabular}{lc||c|c}
\hline & & $\mathcal{G}_{m e a n}(\mathrm{~dB})$ & $\mathcal{G}_{m a x}(\mathrm{~dB})$ \\
\hline \hline IDFT Method & $(\mathrm{T})$ & 0.00076 & 0.00081 \\
& $(\mathrm{E})$ & 0.0035 & 0.0037 \\
\hline \hline Proposed & ARMA(2,2)(T) & 2.5066 & 2.5505 \\
Filter Design & ARMA(2,2)(E) & 2.5068 & 2.5514 \\
& AR(2)(T) & 2.6707 & 2.7247 \\
& AR(2)(E) & 2.6768 & 2.7313 \\
& ARMA(3,3)(T) & 1.9777 & 1.9962 \\
& ARMA(3,3)(E) & 1.9775 & 1.9979 \\
& AR(3)(T) & 2.0924 & 2.173 \\
& AR(3)(E) & 2.1447 & 2.1727 \\
\hline \hline AR Filtering & AR(20)(T) & 2.7 & 2.9 \\
& AR(20)(E) & 2.6 & 2.9 \\
& AR(50)(T) & 0.29 & 0.43 \\
& AR(50)(E) & 0.26 & 0.40 \\
& AR(100)(T) & 0.13 & 0.28 \\
& AR(100)(E) & 0.11 & 0.26 \\
\hline \hline Sum of Sinusoids & 8 Sinusoids(E) & 36.223 & 37.730 \\
& 16 Sinusoids(E) & 4.0264 & 6.4140 \\
& 64 Sinusoids(E) & 0.0211 & 0.0370 \\
& 128 Sinusoids(E) & 0.0027 & 0.0049 \\
\hline \hline
\end{tabular}

IDFT method generally provides closer the highest quality Rayleigh samples. The AR model of [3] provides a more precise match to the desired autocorrelation function as the order of the model used increases. But our proposed filter design method provides same accuracy with much lower order models. As an example, our $\operatorname{ARMA}(3,3)$ fading sequence generator has a significant advantage over $\operatorname{AR}(20)$ generator of [3]. Similar accuracy can be achieved by the WSS sinusoidal generator when a large number of sinusoidal oscillators are used. Proposed ARMA $(3,3)$ fading generator outperforms modified Jakes' generator with 8 and 16 sinusoids by $32 \mathrm{~dB}$ and $2 \mathrm{~dB}$ respectively, while requiring less than one-tenth of the multiplications required by the Jakes' generators with 8 and 16 sinusoids.

The main advantage of our low complexity Rayleigh fading generator is that the samples of the fading sequence can be generated as they are required while achieving the lowest complexity of all the Rayleigh fading generators mentioned. The computational efficiency of the IDFT method brings a cost in storage requirements as all samples are generated using a single IFFT. Our proposed fading generator and the all other generators don't have such a limitation. As provided in Table III, to generate $2^{20}$ samples, IDFT method requires 44040193 real multiplications, our proposed filter design technique via $\operatorname{ARMA}(3,3)$ model requires 12582912 real multiplications, AR(20) model of [5] requires 41943040 multiplications and the improved Jakes' model of [7] requires 356515840 multiplications.

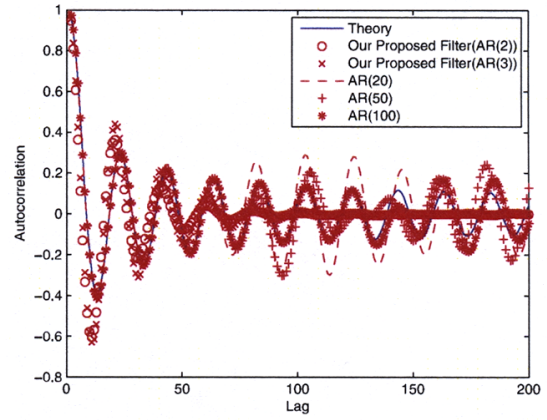

Fig. 3. The empirical autocorrelations for AR method and our proposed model

\section{CONCLUSION}

A low-complexity high performance Rayleigh fading simulator has been proposed. Our proposed $\operatorname{ARMA}(3,3)$ has been compared with improved Jakes' model of [7], AR fading filter approximation of [3], and to the IDFT technique of [5], in terms of performance measures and computational complexity. Our ARMA $(3,3)$ Rayleigh fading generator, outperforms $\mathrm{AR}(20)$ generator of [3], by about $1 \mathrm{~dB}$ in both performance measures provided, while requiring approximately a quarter 


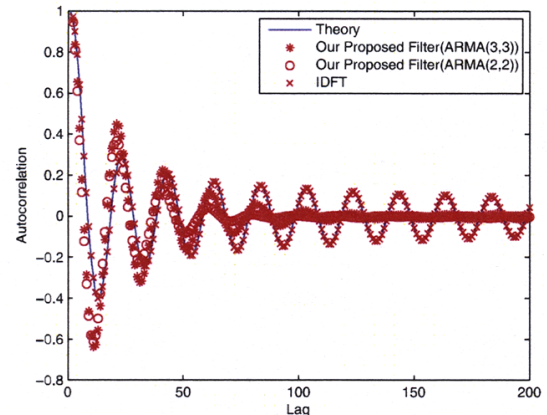

Fig. 4. The empirical autocorrelations for IDFT method and our proposed model

TABLE III

COMPUTATIONAL COMPLEXITY (NUMBER OF REAL MULTIPLICATIONS REQUIRED) COMPARISON TO GENERATE $2^{20}$ SAMPLES

\begin{tabular}{lc||c}
\hline & & $\begin{array}{c}\text { Number of } \\
\text { Real Multiplications }\end{array}$ \\
\hline \hline IDFT Method & & $44 \times 10^{6}$ \\
\hline \hline Proposed Filter & ARMA(2,2) & $8 \times 10^{6}$ \\
Design & AR(2) & $2 \times 10^{6}$ \\
& ARMA(3,3) & $12 \times 10^{6}$ \\
& AR(3) & $6 \times 10^{6}$ \\
\hline \hline AR Filtering & AR(20) & $42 \times 10^{6}$ \\
& AR(50) & $105 \times 10^{6}$ \\
& AR(100) & $210 \times 10^{6}$ \\
\hline \hline Sum of Sinusoids & 8 Sinusoids & $178 \times 10^{6}$ \\
& 16 Sinusoids & $356 \times 10^{6}$ \\
& 64 Sinusoids & $1424 \times 10^{6}$ \\
& 128 Sinusoids & $2848 \times 10^{6}$ \\
\hline \hline
\end{tabular}

of the multiplications required by the AR(20) generator. Similarly, our $\operatorname{ARMA}(3,3)$ fading generator outperforms modified Jakes' generator with 8 and 16 sinusoids by $32 \mathrm{~dB}$ and $2 \mathrm{~dB}$ respectively, while requiring less than one-tenth of the multiplications required by the Jakes' generators with 8 and 16 sinusoids. While the IDFT method of [5] achieves the best performance in terms of the quality measures, it brings a significant cost in storage requirements as all samples are generated using a single IFFT. Thus the IDFT method is undesirable from simulation point of view when the Rayleigh fading samples are generated as they are required. The main advantage of our ARMA $(3,3)$ Rayleigh fading generator is that the samples of the Rayleigh fading sequence can be generated as they are required while achieving the lowest complexity of all the Rayleigh fading generators mentioned.

\section{ACKNOWLEDGMENT}

This research has been supported by EU-FP6 "Marie Curie" International Reintegration Grant, Contract No: 029157.

\section{REFERENCES}

[1] W. C. Jakes Microwave Mobile Communications. Address: Wiley, 1974.
[2] S. Özen, M. D. Zoltowski, "A Fading Filter Approximation to Enable State-Space Modeling and Joint Data/Channel Estimation of (Timevarying) Frequency Selective Channels with Antenna Arrays," in IEEE Circuits and Systems Society Notre Dame Workshop on Wireless Comm. and Networking, South Bend IN, Aug. 2001.

[3] K. E. Baddour and N. C. Beaulieu, "Autoregressive modeling for fading channel simulation," IEEE Trans. on Wireless Commun., , vol. 4, no. 4, pp.1650-1662 July 2005 .

[4] J. I. Smith, "A computer generated multipath fading simulation for mobile radio ," IEEE Trans. on Veh. Technol., , vol. VT-24, pp. 39-40, August 1975.

[5] D. J. Young and N. C. Beaulieu, "The generation of correlated Rayleigh random variates by inverse discrete Fourier transform ," IEEE Trans. on Commun., , vol. 48, no. 7, pp. 1114-1127, July 2000.

[6] D. J. Young and N. C. Beaulieu, "Limitations of sum-of-sinusoids fading channel simulators ," IEEE Trans. on Commun.,, vol. 49, no. 4, pp.699708, Apr.2001.

[7] Y. R. Zheng and C. Xiao, "Improved models for the generation of multiple uncorrelated Rayleigh fading waveforms ", IEEE Commun. Lett. ,vol. 6, no. 6, pp. 256-258, Jun. 2002

[8] D. J. Young and N. C. Beaulieu, " Power margin quality measures for correlated random variates derived from the normal distribution," IEEE Trans. Inf. Theory, vol.49, no. 1, pp.241-252, Jan.2003

[9] P. A. Bello, "Characterization of randomly time-variant linear channels ," IEEE Trans. Commun. Syst. ,vol. CS-11, no. 4, pp.360-393, Dec. 1963.

[10] J. G. Proakis, D. K. Manolakis Digital Signal Processing: Principles, Algorithms and Applications. Address: Prentice Hall, 4th Edition 2007. 